\title{
Powertrain Test Bench System
}

P.M.Fonte

Instituto Superior de Engenharia de Lisboa, Instituto Politécnico de Lisboa Low Carbon Energy Conversion R\&D Group, ISEL, IPL

Lisbon, Portugal ISRC

pfonte@deea.isel.ipl.pt

\section{R. Pereira}

Instituto Superior de Engenharia de Lisboa, Instituto Politécnico de Lisboa Low Carbon Energy Conversion R\&D Group, ISEL, IPL Lisbon, Portugal ISRC

rpereira@deea.isel.ipl.pt

\section{P. Almeida}

Instituto Superior de Engenharia de Lisboa, Instituto Politécnico de Lisboa Low Carbon Energy Conversion R\&D Group, ISEL, IPL

Lisbon, Portugal

palmeida@sa.isel.pt

M.Chaves

Instituto Superior de Engenharia de

Lisboa, Instituto Politécnico de Lisboa

INESC-ID

mchaves@deea.isel.ipl.pt

\author{
R. Luis \\ Instituto Superior de Engenharia de \\ Lisboa, Instituto Politécnico de Lisboa \\ Low Carbon Energy Conversion R\&D \\ Group, ISEL, IPL \\ INESC-ID \\ Lisbon, Portugal \\ rluis@deea.isel.pt
}

\begin{abstract}
In parallel with major automotive manufacturers, some smaller companies are trying to launch their own electric or hybrid-propulsion models. During the development of these prototypes some tests must be done, namely consumption, autonomy, batteries state-of-charge, gradability, among others. The results of these tests can lead changes in design, motorization or battery packages. This paper presents a powertrain test bench system in order to test electrical vehicles. Regarding the automobile sector, and due to EU ruling, it is intended to enable the test bench to apply the Worldwide Harmonized Light Vehicles Test Cycle. Having a laboratorial test bench that can emulate vehicle dynamics under a specific drive cycle will allow small automotive manufacturers to confirm compliance with the mandatory drive cycles during different stages of their development. Nevertheless, other electric vehicles (EV) like boats or trains, can also be tested since the mathematical models of their dynamics are available.
\end{abstract}

Keywords- electric propulsion, drive cycles, hardware-inthe-loop

\section{INTRODUCTION}

Automotive manufacturers must create solutions to achieve the best performance indicators to attract their clients. These indicators can be comfort, security, power, torque or ecological and economic indicators, such as emissions, durability, and autonomy, among others. Besides the security tests, many other must be performed, such as, exhaust gas emissions ( $\mathrm{CO}, \mathrm{CO}_{2}, \mathrm{NO}_{\mathrm{x}}$ and THC), as well as for the assessment of particulate emissions, and fuel consumption which must meet whole limits defined by the legislators [1]. Thus, it is vital to implement measuring systems in order to obtain the largest amount of information about the drive systems. To achieve these goals some test benches have been developed, namely chassis dynamometers for dynamic tests and portable measuring systems for emissions.
Notice that some of these tests are standard and mandatory to approve and distinguish the vehicles between groups. In the specific case of dynamic test benches, it is possible to define the operation parameters of the vehicle under study and simulate the dynamic of accelerations and braking. It is also possible to create specific drive cycles and even driver's driving characteristics [1].

With the increased demand for battery EV the main concern is no longer emissions, the focus has shifted to driving performances and autonomy. The aim of this work is to propose a battery EV powertrain test bench, prepared to rehearse several drive cycles and conclude about vehicle consumption, autonomy and driving dynamics, even in an early development stage of vehicle project.

This paper is organized as follows: section II presents an overview about standard drive cycles; section III describes and characterizes the developed test bench; the implemented mathematical model is stated in section IV; example results and performance analysis are presented in Section $\mathrm{V}$ and conclusions are given in section VI.

\section{DRIVE CYCLES}

In the European Union, certification tests are mandatory for all light duty vehicles and for heavy duty vehicles with more than 3.5 Ton. However, and because the composition of exhaust gases is diverse, the best way to uniform the tests procedures is to perform them in a laboratorial environment, using a patterned procedure.

This allows an adequate replication of those tests. The data is collected for different pre-defined drive cycles, which intends to emulate the real vehicle behavior [3]. Drive cycle analyses allows inferring of the vehicle's consumption and their corresponding pollutant emissions in a standardized way, enabling an adequate comparison between vehicles. 
A graphical drive cycle representation generally shows vehicle velocity vs time, or vehicle velocity vs torque.

There are several drive cycles defined by different countries and organizations to be applied in light vehicles, heavy duty vehicles and motorcycles [4], [5]. Focusing on light duty vehicles, the New European Drive Cycle (NEDC), used to be applied in European countries, the JC08 was applied in Japan and the Federal Test Procedure (FTP 75) was applied in the USA. The NEDC showed four urban cycle segments, characterized by a low vehicle velocity, low torque and low exhaustion gases temperature. In addition, an extra-urban segment was used to characterize a higher vehicle velocity and a more demanding driving type. JC08 characterized urban driving with congested traffic, including low velocity with frequent accelerations and slowdowns. FTP-75 was characterized by a transient cycle defined using real data collected in Los Angeles [4]. A graphic representation of these drive cycles is detailed in [5], [6]. Regarding only European drive cycles, about 10 different types can be found in [5]. This shows that the existing drive cycles were significantly different, which was a challenge in terms of data and procedure standardization. In order to respond to this problem a Worldwide Harmonized Light Duty Driving Test Cycle (WLTC), has been developed and used as a basis for certified tests procedures since 2014 [4]. In 2017, European commission decided to replace NEDC by WLTC [6], [7].

The WLTC, was developed using real simulation data collected in 5 different regions: Europe/Switzerland, USA, India, Korea and Japan. It also includes a wide range of light duty vehicles with different engines, different weight-power ratios, different road types (urban, rural, highway) and different traffic status (rush hour, light traffic and weekend). From this development resulted the following procedures: i) WLTC CL1 (v.2.0) for class 1 vehicles; ii) WLTC CL2 (v.2.0) for class 2 vehicles; iii) WLTC CL3 (v5.1) for class 3 vehicles with less than $120 \mathrm{~km} / \mathrm{h}$ maximum velocity; iv) WLTC CL3 (v5.3) for class 3 vehicles with more than 120 $\mathrm{km} / \mathrm{h}$ maximum velocity. Detailed data about the previously referred tests procedures can be found in [5], based on the ARTEMIS parameters which allows to characterize vehicles kinematics for each test procedure.

The application of the WLTC in EV is recent, nevertheless data collected from hybrid vehicles, shows that the change from the NEDC to the WLTC, has contributed for a better validation between performed tests and real vehicle behavior. This also, allowed to demonstrate the efficiency of hybrid vehicles efficiency in $\mathrm{CO}_{2}$ emissions reduction. Because of the performed studies and analysis, it is possible to infer that the WLTC is adequate to be applied to EV and, in this case, the EV performance mainly depends on the energy management system performance for each tested vehicle.

In case of an EV, the most important test is the range. For instance, the ratio between the battery package weight and the EV autonomy is very sensible, since the extra added weight can compromise the total range of the vehicle.

Nowadays the project of an EV starts by its dimensioning in specific software which can calculate the value of the mass, frontal area, drag resistance, rolling resistance, and overall efficiency. It is also possible to calculate theoretically the consumption and the autonomy of the designed vehicle.

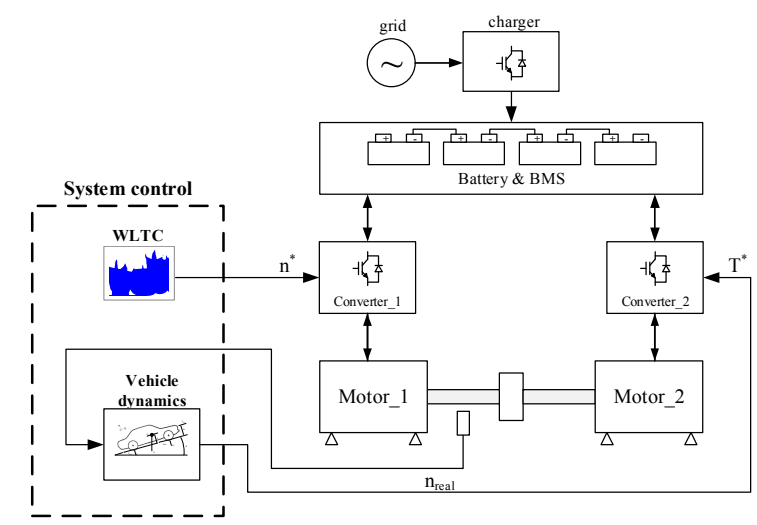

Fig. 1. Overall system

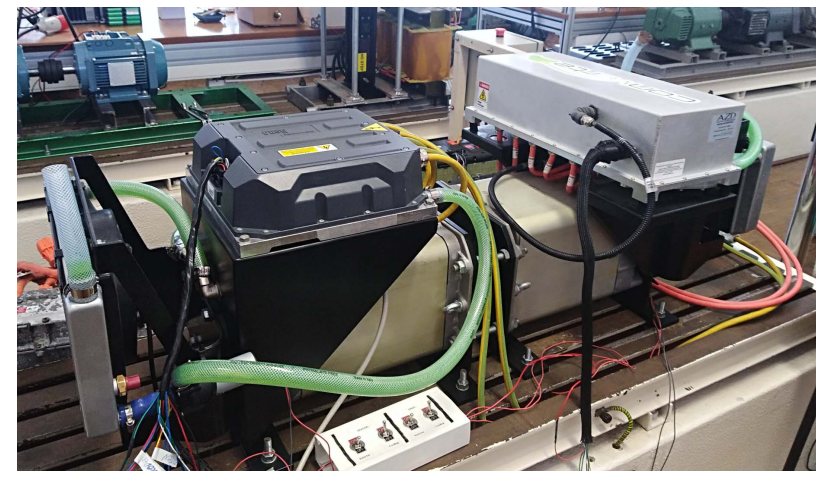

Fig. 2. Laboratorial implementation

Once the vehicle is constructed, additional tests are done in order to validate the theoretical analysis. Those tests include the cost-down method and wind tunnel, to determine rolling resistance and aerodynamic drag coefficients. These values will be reproduced on a chassis dynamometer dynamic bench to validate the simulated ones.

\section{SYSTEM CHARACTERIZATION}

\section{A. Test Bench Hardware}

The test bench is composed by two identical three-phase squirrel-cage induction machines with $45 \mathrm{~kW} \mathrm{S2} 30 \mathrm{~min}$ and two inverters and a $2^{\text {nd }}$ life automotive battery pack with 16 $\mathrm{kWh}$, in Figure 1 the overall system is depicted. Motor 1 and converter_1, are the power train to be tested while motor_2 and converter_2 emulate the vehicle dynamics and the resistant forces applied to the vehicle body, it acts as dynamic brake. Both sets are fed by the same battery pack.

In Figure 2 the laboratorial implementation of the power bench in development is depicted.

\section{B. Test Bench Command}

A Matlab ${ }^{\circledR}$ based software is used to control both test bench powertrains, via CAN-BUS. Within a fixed step time, a speed reference defined by the drive cycle under study is sent to the inverter_1 which drives Motor_1, as depicted in Figure 3. With the measured speed, the equivalent resistant force applied to the vehicle is calculated and converted into torque reference for the converter_2, as shown in figure 4. This torque reference represents the rolling and dragging resistances, the force of vehicle inertia during the accelerations and decelerations, as well as the gradability of the drive cycle. 


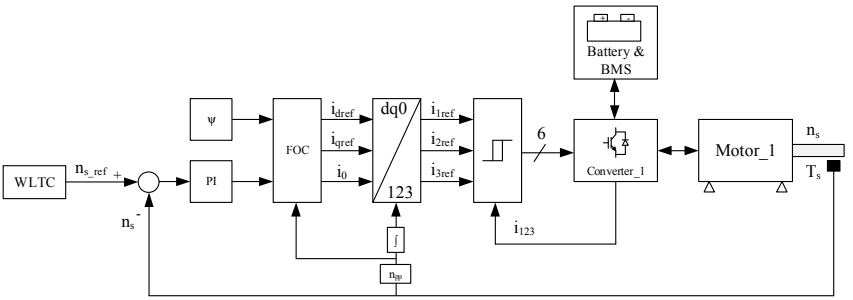

Fig. 3. Power train control model

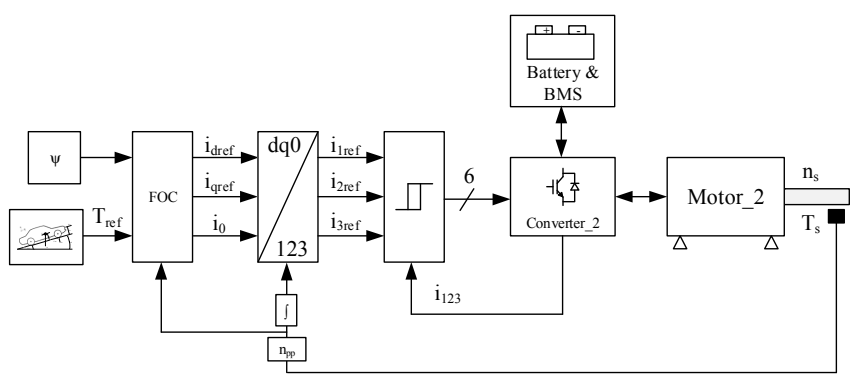

Fig. 4. Vehicle dynamic control

\section{SYSTEM MODEL}

From Newton's laws, a vehicle dynamic equation is determined by all forces acting on the vehicle [8]. In (1) $F_{x}$ represents the tractive force exerted by the tire surface in contact with the road, which will propel the vehicle. This force results from the torque produced by the motor and transferred to the wheel through a transmission and a final drive. Also, in (1), $F_{J}$ is the inertia force, $F_{I}$ the gradability force, $F_{R R}$ the rolling force and $F_{D A}$ the aerodynamics force.

$$
F_{x}+F_{J}+F_{I}+F_{R R}+F_{D A}=0
$$

Equation (2) describes the inertial resistant force $\left(F_{J}\right)$ which depends on the mass and speed changes. Although it is zero at constant speed, it has a crucial role in the system dynamic, namely during starts and speed transitions. The inertial resistance during accelerations does not depend only on vehicle weight but also on rotational masses.

They include all the accelerated rotational masses, starting form crank system parts to vehicle wheels [2]. The parameter $\delta$ is the correction factor associated to the rotating masses. Due to the inexistence of crank system and flywheel with clutch mechanism which has the main contribution to $\delta$, its value is considered one in this case.

$$
F_{J}=m \delta \frac{d v}{d t}
$$

The angle of the road also influence the force applied on the vehicle by (3), being positive under a positive slope and thus breaking the vehicle or accelerating it on downhills.

$$
F_{I}=m g \sin (\theta)
$$

The rolling resistance force can be modeled by (4). Where $f_{r}$ is rolling resistance coefficient, $m$ and $g$, are respectively the vehicle mass and the gravity force, while $\theta$ is the slope.

$$
F_{R R}=f_{r} m g \cos (\theta)
$$

However, the value of rolling resistance coefficient is not trivial to determine since it depends on tire pressure, speed, floor irregularities, weight force application point, and tire lateral deflection, among others. Thus, the rolling resistance coefficient is empirically calculated for light-duty passenger vehicles circulating in asphalt by (5), with speed $V$ in $\mathrm{Km} / \mathrm{h}$. This expression is considered valid up to approximately 128 $\mathrm{km} / \mathrm{h}[8]$.

$$
f_{r} \cong 0,01\left(1+\frac{V}{160}\right)
$$

The aerodynamic resistance force (drag force) is given by (6) where $\rho$ is the air density and $A_{f}$ is the frontal area of the vehicle. The aerodynamic resistance coefficient $C_{D}$ can be calculated experimentally in a wind tunnel, or in a virtual wind tunnel with specific software for fluid dynamics analysis. However, there are indicative values for certain classes of vehicles, which can serve as first approach to the calculus. Finally, the speed results from the subtraction between vehicle and wind speeds. The wind speed $v_{w}$ is considered positive when it is in the same moving direction of vehicle and negative when it is opposite to the vehicle moving direction.

$$
F_{D A}=\frac{1}{2} \rho C_{D} A_{f}\left(v-v_{w}\right)^{2}
$$

There are two more forces that can be considered in the model, namely the lateral and elevation forces (7) and (8).

$$
F_{L}=\frac{1}{2} \rho C_{L}(\beta) A_{f} v^{2}
$$

Lateral force coefficient $C_{L}$ depends on the angle $\beta$ between the vehicle movement direction and the wind speed. In (8) $C_{E}$ is the elevation coefficient.

$$
F_{E}=\frac{1}{2} \rho C_{E} A_{f} v^{2}
$$

As conclusion, at low speed the rolling resistance force is predominant while at high speed is the aerodynamic one. The inertial resistance force will act only during the speed transitions.

The instantaneous power and overall energy changed during the drive cycles are calculated using (9) and (10).

$$
\begin{gathered}
p=F v \\
W=\int p d t
\end{gathered}
$$

\section{CASE STUdy}

The proposed test bench will be tested under the WLTC class 2 .

This drive cycle is proposed for vehicles with a mass/power ratio between 22 and 34, traveling a distance of 14664 meters in 1477 seconds. In this case study the gradient of the road is considered null, meaning that angle $\theta$ is zero.

In table I the parameters chosen for the vehicle under study are shown. 
TABLE I. VEHICLE CHARACTERISTICS

\begin{tabular}{|l|c|}
\hline \multicolumn{1}{|c|}{ Parameters } & Value \\
\hline Battery capacity & $10 \mathrm{kWh}$ \\
\hline Mass $(m)$ & $830 \mathrm{~kg}$ \\
\hline Rolling resistance coeficient $\left(f_{r}\right)$ & 0,01 \\
\hline Drag coefficient $\left(C_{D}\right)$ & 0,3 \\
\hline Frontal area $\left(A_{f}\right)$ & $1,88 \mathrm{~m}^{2}$ \\
\hline Transmission ratio $\left(t_{r}\right)$ & 7 \\
\hline Transmission efficiency $(\eta)$ & $97 \%$ \\
\hline Tire & $155 / 70 \mathrm{R} 13$ \\
\hline Air density $(\rho)$ & 1,225 \\
\hline
\end{tabular}

In WLTC the speed reference is given in $\mathrm{km} / \mathrm{h}$. As the converter only accept references in revolution per minute (rpm) referred to motor's shaft $\left(n_{s \text { ref }}\right)$, the speed reference has to be converted by (11),

$$
n_{S_{-} r e f}=\frac{60 t_{r}}{2 \pi 3.6 r_{w}} v_{r e f}
$$

where $t_{r}$ is the transmission ratio, $r_{w}$ is the wheel radius and $v_{\text {ref }}$ is the linear reference speed of the vehicle in $\mathrm{ms}^{-1}$. The wheel radius, will depend on the chosen tire, and is calculated by (12).

$$
r_{w}=\frac{(w \times h) / 100+R \times 25.4}{1000}
$$

In (12) $w$ is the tire width (in millimeters) and $h$ is the aspect ratio in percentage of $w$ while $R$ is the radius of car rim (in inches).

Once the speed reference is send to converter_1, the actual speed is measured and the total tractive force $F_{x}$ is calculated from (2) to (8). The load reference torque is calculated as (13).

$$
T_{r e f}=\frac{F_{x} r_{w}}{r_{t} \eta}
$$

As the power converters demand for a certain cadency of CAN messages, the references are recalculated in order to send values with $100 \mathrm{~ms}$ intervals.

\section{A. Drive cycle simulation}

In Figure 5 the reference speed, as well as the measured are depicted. It is verified that measured speed has a good fitting to the reference. To better evaluate in figure 6 a zoom defined by the rectangle is depicted. In Fig 6 is clearly demonstrated the capacity of the motor to follow the drive cycle reference even with the dynamic imposed by the vehicle. It is also possible to verify that measured speed becomes negative when the reference is set to zero. This happen because there is no brake system, pedal and the hill hold parameter of the converter 1 is disabled and the existing of some delay of the load torque imposed by the vehicle. Thus, when the reference is set to zero the measured speed is slightly negative returning to zero after few seconds. This difference is marginal and can be avoided with a convenient parametrization of the converter.

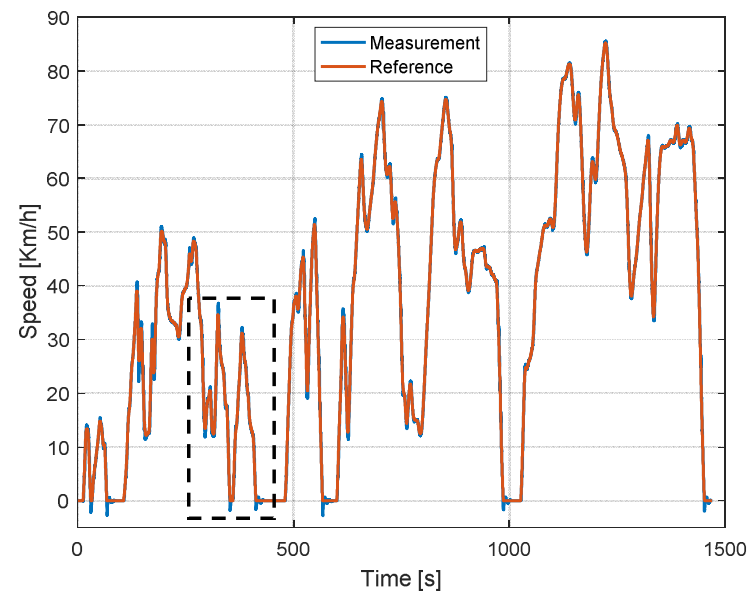

Fig. 5. Speed reference $v s$ measured speed

In Figure 7 the torque reference calculated by (13) and the measured are shown. Once again there is a good matching between the two signals meaning that the expected dynamic is being applied to the power train.

Although the dynamic of a vehicle is relatively slow, in Figure 8 rapid variations of the torque are depicted. The cadency of the torque references generated by the software is 10 times per second, which together with the fast dynamic of the converters and electric motors, lead to the fast variations of the reference and measured. Nevertheless, this does not affect the final values neither the overall conclusions.

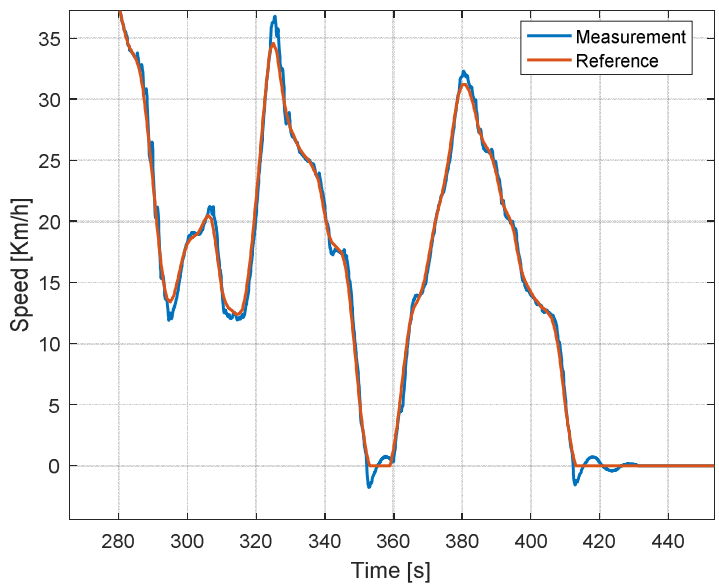

Fig. 6. Speed reference $v s$ measured speed (zoom)

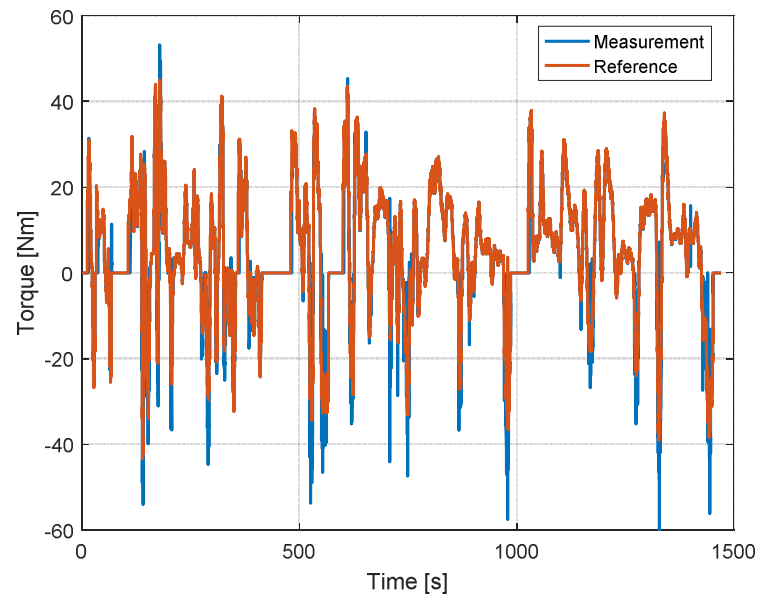

Fig. 7. Torque reference $v s$ measured torque 


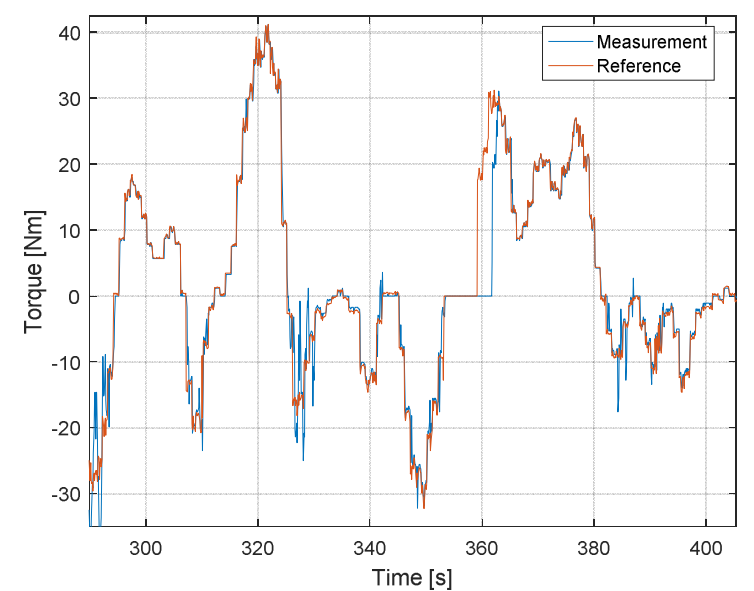

Fig. 8. Torque reference $v s$ measured torque (zoom)

Figure 9 and 10 depicts, respectively the power and the accumulated energy consumption of the power train during the drive cycle. The behavior of the graph allows to understand when the motor is consuming energy and when is regenerating, during the deceleration periods.

At the end of the drive cycle the accumulated energy consumption was $0,804 \mathrm{kWh}$. Considering the battery capacity of $10 \mathrm{kWh}$ and the distance of the drive cycle, $14644 \mathrm{~m}$ is possible to calculate the autonomy of the tested vehicle, $182 \mathrm{Km}$.

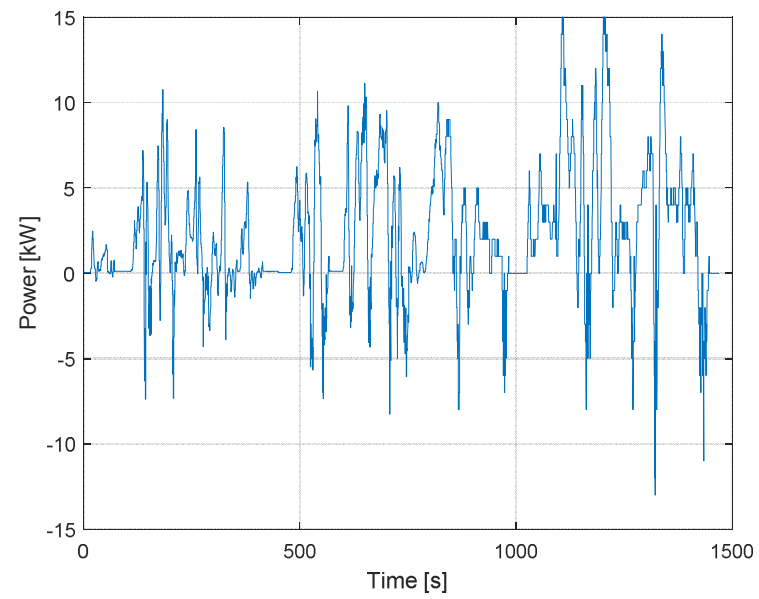

Fig. 9. Power flow during the drive cycle

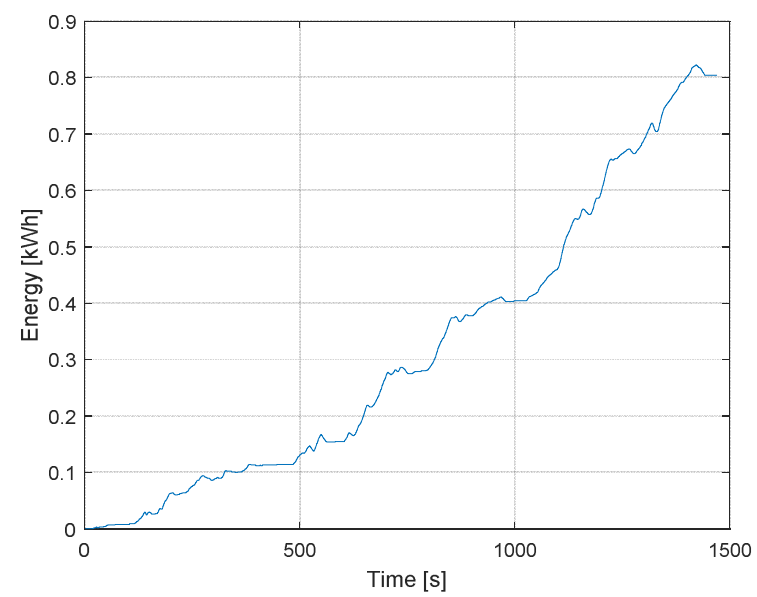

Fig. 10. Energy consumed by the power train

\section{CONCLUSIONS}

The proposed test bench resulted from a design that allow to emulate several drive cycles in academic laboratorial environment. The implemented mechanical structure enables to use the designed system to perform several dynamic tests for different vehicles whose characteristics and parameters are suitable for the test bench secure performance.

The test bench provides a fast and cost-effective way in comparison with on-road testing or chassis dynamometer test benches. It also allows repeating the same test conditions with different configurations of powertrains, electric motor, batteries, inverter, and gears, cooling systems, among others, in order to achieve the best configuration.

This test bench also allows testing specific vehicles in specific circuits, adding different gradability and loads, to study the capacity of the vehicle to comprise the drive cycle. This test bench can be used as a first approach to project new vehicles or reconfigure existing ones. However, it must be kept in mind that this testing cannot reflect precisely the real-world driving conditions once there are always some mathematic simplifications. For instance, the driving resistance forces applied to the vehicle on the test bench are derived from computational simulations or cost-down test done under different conditions from those applied in the test making it difficult to accurately reflect resistance in the real world. Nevertheless, the results of these tests can evince the direction for vehicle developments in order achieve important conclusions about the design and performances of EV. This facility is not limited to road EV's could be used to test other kind of vehicles like boats or trains since the mathematic models of its dynamic are available.

\section{ACKNOWLEDGMENT}

The authors would like to acknowledge Instituto Politécnico de Lisboa for the research grants that enabled the prosecution of this research.

\section{REFERENCES}

[1] L. Rymaniak et Al., Comparative test of an internal combustion engine ecological indicators in real operating conditions and on a dynamic engine dynamometer, 2018 IOP Conference Series: Materials, Science and Engineering Issue 4, Vol. 421, 2018.

[2] Ubysz, Aleksander, Problems of rotational mass in passenger vehicles, Journal of Problems of rotational mass in passenger vehicles", Vol 5, issue 1, pages 33-40,2010.

[3] Q. Shi,Y. Zheng,R. Wang,Y. Li, The study of a new method of driving cycles construction, Procedia Engineering 16 (2011) $79-87$.

[4] M. Tutianu, et Al., Development of a Worldwide harmonized light duty drivind test cycle (WLTC) - Technical Report, (2013) UN/ECE/WP.29/GRPE/WLTP-IG, DHC subgroup, 1-103.

[5] T.J.Barlow, S. Latham, I.S. McCrae, P.G. Boulter, A reference book of driving cycles for use in the measurements of road vehicle emissions, Published project report PPR354, Version 3, TRL limited, (2009), 1284.

[6] Dieselnet, https://www.dieselnet.com/standards/cycles/jp_jc08.php, acessed on 2018-12-26.

[7] C. Cubito et Al., Impact of Different Driving Cycles and Operating Conditions on CO2 Emissions and Energy Management strategies of a Euro-6 Hybrid Electric Vehicle, Energies ,doi:10.3390/en10101590, 10 (2017) 1-18.

[8] M. Ehsani, Y. Gao, S. Longo, K. Ebrahimi, "Modern Electric, Hybrid Electric, and Fuel Cell Vehicles", $3^{\text {rd }}$ edition, CRC Press, 2018, ISBN: 978-1-4987-6177-2. 